ESAIM: COCV $22(2016) 862-871$

DOI: $10.1051 / \mathrm{cocv} / 2015034$
ESAIM: Control, Optimisation and Calculus of Variations

www.esaim-cocv.org

\title{
STRICT CONVEXITY AND THE REGULARITY OF SOLUTIONS TO VARIATIONAL PROBLEMS*
}

\author{
Arrigo Cellina ${ }^{1}$
}

Abstract. We consider the problem of minimizing

$$
\int_{\Omega}[L(\nabla v(x))+g(x, v(x))] d x \quad \text { on } \quad u^{0}+W_{0}^{1,2}(\Omega)
$$

where $\Omega$ is a bounded open subset of $\mathbb{R}^{N}$ and $L$ is a convex function that grows quadratically outside the unit ball, while, when $|\nabla v|<1$, it behaves like $|\nabla v|^{p}$ with $1<p<2$. We show that, for each $\omega \subset \subset \Omega$, there exists a constant $H$, depending on $\omega$ but not on $p$, such that both

$$
\|\nabla u\|_{W^{1,2}(\omega)} \leq H \text { and }\left\|\frac{\nabla u}{|\nabla u|^{2-p}}\right\|_{W^{1,2}(\omega)} \leq \frac{H}{(p-1)^{2}}
$$

in particular, for every $i=1, \ldots N$, we have $\max \left\{\frac{\left|u_{x_{i}}\right|}{|\nabla u|^{2-p}},\left|u_{x_{i}}\right|\right\} \in W_{l o c}^{1,2}(\Omega)$.

Mathematics Subject Classification. 49K10.

Received December 12, 2014.

\section{INTRODUCTION}

This paper is concerned with the regularity properties of solutions to variational problems and, more precisely, with their properties of higher differentiability. We consider the problem of minimizing

$$
\int_{\Omega}[L(\nabla v(x))+g(x, v(x))] \mathrm{d} x \quad \text { on } \quad w^{0}+W_{0}^{1,2}(\Omega)
$$

where $L$ is a convex function and $\Omega$ a bounded open subset of $\mathbb{R}^{N}$. We wish to explore the effect of an increase of the strict convexity of the Lagrangian, with respect to the variable gradient, on the regularity of the solution; more precisely, we consider a problem where $L$ grows quadratically outside the unit ball, while, when $|\nabla v|<1$,

Keywords and phrases. Regularity of solutions, higher differentiability, strict convexity.

* This work was partially supported by INDAM-GNAMPA.

1 Dipartimento di Matematica e Applicazioni, Università degli Studi di Milano-Bicocca, Via R. Cozzi 53, 20125 Milano, Italy. arrigo.cellina@unimib.it 
it behaves like $|\nabla v|^{p}$ with $1<p<2$; hence, near the origin, the norm of the matrix of the second derivatives of $L$ diverges, making the problem very strictly convex when $|\nabla v|$ is small. Our Theorem 2.2 below describes how this increasing in the strict convexity of $L$ affects the higher differentiability of the solution $u$, when $|\nabla u|$ is small.

Regularity results in the sense of $u$ being in $C^{1, \alpha}$ for $L(\xi)=|\xi|^{p}$ with $p>1$ have been proved by Uhlenbeck [8], Lewis [6] and Tolksdorf [7] for $g=0$ and by Di Benedetto [2], Acerbi and Fusco [1] in the general case; very recently functionals with different conditions on $\{|\xi|>1\}$ and on $\{|\xi|<1\}$, (with $g=f u$ ) have been considered by Colombo and Figalli [5] and the regularity $C^{1, \alpha}$ of the solution established; these results and techniques are different from ours.

\section{Statement of the Theorem}

The integrand $L$ of (1.1) is described as follows: for some $1<p<2$, we shall consider the function

$$
l(t)= \begin{cases}\frac{1}{2}|t|^{2}+1 & \text { for }|t| \geq 1 \\ \frac{1}{p}|t|^{p}+\frac{3}{2}-\frac{1}{p} & \text { for }|t| \leq 1\end{cases}
$$

and set $L(\xi)=l(|\xi|)$. We have that, calling $H_{L}(\xi)$ the matrix of second derivatives of $L$ computed at $\xi$,

$$
H_{L}(\xi)= \begin{cases}(p-2)|\xi|^{p-4} \xi \otimes \xi+|\xi|^{p-2} I & \text { for }|\xi|<1 \\ I & \text { for }|\xi|>1\end{cases}
$$

so that $z^{T} H_{L}(\xi) z \geq|z|^{2}$ for all $\xi$, while $\left|H_{L}(\xi)\right| \rightarrow \infty$ as $|\xi| \rightarrow 0$.

The assumptions on $g$ are:

\section{Assumption 2.1.}

i) There exist $\tau \in L^{1}(\Omega)$ and a non-negative $\lambda_{g} \in L_{\text {loc }}^{2}(\Omega)$ such that for a.e. $x \in \Omega$ and every $u$, we have $g(x, u) \geq \tau(x)-\lambda_{g}|u|$.

ii) There exist non-negative $\lambda_{2} \in L_{\mathrm{loc}}^{2}(\Omega)$ and $\lambda_{\infty} \in L_{\mathrm{loc}}^{\infty}(\Omega)$, such that $\left|g_{u}(x, u)\right| \leq \lambda_{2}(x)+\lambda_{\infty}(x)|u|$.

Functions like $g(x, u)=\lambda_{2}(x) u$ or $g(x, u)=\left(\sin \left(x_{1}\right) u\right)^{2}$ satisfy Assumption 2.1.

The map $l$, and the map $l_{r}$ to be defined, are not really $C^{2}$ everywhere, but their gradients are Lipschitzian, and, by a simple modification of results that go back to [3], one proves that a solution $u$ to the problem of minimizing (1.1), with $L$ and $g$ described above, is such that $\nabla u \in W_{\text {loc }}^{1,2}(\Omega)$.

The purpose of this paper is to prove the following result:

Theorem 2.2. Let $\Omega$ be a bounded open subset of $\mathbb{R}^{N}$, let $l$ be as in (2.1) and let $g$ satisfy Assumption 2.1. Then, there exist $u$, a solution to the Euler-Lagrange equation, i.e. such that

$$
\int_{\Omega}\left[\langle\nabla L(\nabla u(x)), \nabla \eta(x)\rangle+g_{u}(x, u(x)) \eta(x)\right] \mathrm{d} x=0
$$

for every $\eta \in C_{c}^{1}(\Omega)$, and, for each $\omega \subset \subset \Omega$, a constant $H$, depending on $\omega$ but not on $p$, such that both

$$
\|\nabla u\|_{W^{1,2}(\omega)} \leq H \text { and }\left\|\frac{\nabla u}{|\nabla u|^{2-p}}\right\|_{W^{1,2}(\omega)} \leq \frac{H}{(p-1)^{2}}
$$

in particular, for every $i=1, \ldots N$, we have $\max \left\{\frac{\left|u_{x_{i}}\right|}{|\nabla u|^{2-p}},\left|u_{x_{i}}\right|\right\} \in W_{\mathrm{loc}}^{1,2}(\Omega)$. 
Under some additional assumptions, mainly when $g$ is convex in the variable $v$, a solution to the Euler-Lagrange equation is actually a solution to the minimization problem (1.1).

The additional regularity of the solution, provided by Theorem 1, is actually lost in the limit as $p \rightarrow 1$, as the statement of Theorem 2.2 itself suggests. In fact, the limit problem consists in minimizing (1.1) where $L(\xi)=l_{*}(|\xi|)$ with

$$
l_{*}(t)= \begin{cases}\frac{1}{2}|t|^{2}+1 & \text { for }|t| \geq 1 \\ |t|+\frac{1}{2} & \text { for }|t| \leq 1\end{cases}
$$

here we have at once that $l_{*}^{\prime \prime}(0)=\infty$ while $l_{*}^{\prime \prime}(t)=0$ for $0<|t|<1$. When $g(x, u)=u$, a (radial) solution to problem (1.1) is

whose gradient is

$$
u_{*}(x)= \begin{cases}0 & \text { for }|x| \leq 1 \\ \frac{1}{2}\left(|x|^{2}-1\right) & \text { for }|x| \geq 1\end{cases}
$$

$$
\nabla u_{*}(x)= \begin{cases}0 & \text { for }|x|<1 \\ x & \text { for }|x|>1\end{cases}
$$

so that the gradient is discontinuous along $|x|=1$, preventing $\nabla u_{*}$ from being a Sobolev function.

\section{Proof of Theorem 2.2}

We shall use the following notations. The measure of $A \subset \mathbb{R}^{N}$ is $|A| ; a^{T}$ is the transpose of $a$; for a fixed coordinate direction $e_{s}$, we set $\delta_{h e_{s}} u$ to be the difference quotient of the function $u$, defined by $\delta_{h e_{s}} u(x)=$ $\frac{u\left(x+h e_{s}\right)-u(x)}{h}$. For a variation $\eta$ to be defined, $D_{\eta}$ is such that $|\nabla \eta(x)| \leq D_{\eta}$.

For the proof of the main result we shall need $l_{r}$, a regularization of $l$, defined to be

$$
l_{r}(t)= \begin{cases}\frac{1}{2} r^{p-2} t^{2}+\left(\frac{1}{p}-\frac{1}{2}\right) r^{p}+\frac{3}{2}-\frac{1}{p} & \text { for }|t| \leq r \\ l(t) & \text { otherwise }\end{cases}
$$

so that

$$
l_{r}^{\prime}(t)= \begin{cases}r^{p-2} t & \text { for } 0 \leq t \leq r \\ l^{\prime}(t) & \text { otherwise }\end{cases}
$$

We have that $l_{r}^{\prime}$ is continuous and increasing, hence $l_{r}$ is convex; moreover, for $t \notin\{r, 1\}, l_{r}^{\prime \prime}(t)$ exists and

$$
l_{r}^{\prime \prime}(t)= \begin{cases}r^{p-2} & \text { for }|t|<r \\ (p-1)|t|^{p-2} & \text { for } r<|t|<1 \\ 1 & \text { otherwise. }\end{cases}
$$

In particular, $l_{r}^{\prime}$ is (globally) Lipschitzian with constant $r^{p-2}$. Set $L_{r}(\xi)=l_{r}(|\xi|)$ so that $\nabla L_{r}$ is Lipschitzian with Lipschitz constant $r^{p-2}$. In addition, we have that $\nabla L_{r} \rightarrow \nabla L$ uniformly as $r \rightarrow 0$.

Besides Problem 1.1, we shall also consider the problem of minimizing

$$
\int_{\Omega}\left[L_{r}(\nabla v(x))+g(x, v(x))\right] \mathrm{d} x \quad \text { on } \quad w^{0}+W_{0}^{1,2}(\Omega)
$$

and call $u^{r}$ its solution. By known regularity results, the function $u^{r}$ is in $W_{\text {loc }}^{2,2}(\Omega)$.

Lemma 3.1. Let $\Omega$ and $g$ as in Theorem 2.2 ; let $u^{r}$ be a solution to the minimization of $(3.2)$; let $\phi \in W^{1,2}(\Omega)$ with support compactly contained in $\Omega$. Then, for $s=1, \ldots, N$, we have

$$
\int_{\Omega}\left\langle\frac{\mathrm{d}}{\mathrm{d} x_{s}} \nabla L_{r}\left(\nabla u^{r}\right), \nabla \phi\right\rangle=\int_{\Omega} g_{u}\left(\cdot, u^{r}\right) \phi_{x_{s}}
$$


Proof.

a) First, we claim that the map $\nabla L_{r}\left(\nabla u^{r}\right)$ is in $W^{1,2}(\Omega)$; we have that $\nabla L_{r}(\xi)=l_{r}^{\prime}(|\xi|) \frac{\xi}{|\xi|}$ and that $\left|\nabla u^{r}\right|$ is in $W^{1,2}(\Omega)$, with $\frac{\mathrm{d}}{\mathrm{d} x_{i}}\left|\nabla u^{r}\right|=\left\langle\frac{\nabla u^{r}}{\left|\nabla u^{r}\right|}, \nabla u_{x_{i}}^{r}\right\rangle$. The map

$$
\frac{l_{r}^{\prime}(t)}{t}= \begin{cases}r^{p-2} & \text { for } 0 \leq|t| \leq r \\ |t|^{p-2} & \text { for } r \leq|t| \leq 1 \\ 1 & \text { for }|t| \geq 1\end{cases}
$$

is (uniformly) Lipschitzian and it is not differentiable only at at $|t|=r$ and $|t|=1$; then, as it is known, $x \rightarrow \frac{l_{r}^{\prime}\left(\left|\nabla u^{r}(x)\right|\right)}{\left|\nabla u^{r}(x)\right|}$ is a Sobolev function with

$$
\begin{aligned}
\frac{\mathrm{d}}{\mathrm{d} x_{i}} \frac{l_{r}^{\prime}\left(\left|\nabla u^{r}(x)\right|\right)}{\left|\nabla u^{r}(x)\right|} & =\left[\left(\frac{l_{r}^{\prime}(t)}{t}\right)^{\prime} \circ\left|\nabla u^{r}(x)\right|\left\langle\frac{\nabla u^{r}}{\left|\nabla u^{r}\right|}, \nabla u_{x_{i}}^{r}\right\rangle\right. \\
& = \begin{cases}0 & \text { for }\left|\nabla u^{r}(x)\right| \leq r \text { or }\left|\nabla u^{r}(x)\right| \geq 1 \\
(p-2)\left|\nabla u^{r}(x)\right|^{p-3}\left\langle\frac{\nabla u^{r}}{\left|\nabla u^{r}\right|}, \nabla u_{x_{i}}^{r}\right\rangle & \text { otherwise. }\end{cases}
\end{aligned}
$$

Then

$$
\frac{\mathrm{d}}{\mathrm{d} x_{i}}\left[\frac{l_{r}^{\prime}\left(\left|\nabla u^{r}(x)\right|\right)}{\left|\nabla u^{r}(x)\right|} \cdot \nabla u^{r}(x)\right]=\frac{l_{r}^{\prime}\left(\left|\nabla u^{r}(x)\right|\right)}{\left|\nabla u^{r}(x)\right|} \nabla u_{x_{i}}^{r}(x)+\left(\frac{\mathrm{d}}{\mathrm{d} x_{i}} \frac{l_{r}^{\prime}\left(\left|\nabla u^{r}(x)\right|\right)}{\left|\nabla u^{r}(x)\right|}\right) \nabla u^{r}(x) .
$$

Both terms above are in $L_{\text {loc }}^{2}(\Omega)$ : in fact, $\frac{l_{r}^{\prime}(t)}{t}$ is bounded and, from (3.3), the absolute value of the second term is at most $\left|\nabla u_{x_{i}}\right|$. Hence, $\nabla L^{r}\left(\nabla u^{r}\right)$ is in $W_{\text {loc }}^{1,2}(\Omega)$.

b) Under the assumptions of the Lemma, the Euler-Lagrange equation holds for $u^{r}$ in the sense that for $\psi \in W_{0}^{1,2}(\Omega)$ we have

$$
\int_{\Omega}\left[\left\langle\nabla L_{r}\left(\nabla u^{r}\right), \nabla \psi\right\rangle+g_{u}(x, u) \psi\right] \mathrm{d} x=0 .
$$

For $h$ sufficiently small, consider the variation $\psi=\delta_{-h e_{s}} \phi$ to obtain

$$
\int_{\Omega}\left\langle\frac{\nabla L^{r}\left(\nabla u^{r}\left(x+h e_{s}\right)\right)-\nabla L^{r}\left(\nabla u^{r}(x)\right)}{h}, \nabla \phi(x)\right\rangle \mathrm{d} x=\int_{\Omega} g_{u}\left(x, u^{r}(x)\right) \frac{\phi\left(x-h e_{s}\right)-\phi(x)}{-h} \mathrm{~d} x .
$$

Since $\nabla L^{r}\left(\nabla u^{r}\right)$ is in $W_{\text {loc }}^{1,2}(\Omega)$, the family $\left(\frac{\nabla L^{r}\left(\nabla u^{r}\left(x+h e_{i}\right)\right)-\nabla L^{r}\left(\nabla u^{r}(x)\right)}{h}\right)_{h}$ is bounded in $L_{\text {loc }}^{2}(\Omega)$ and we can assume the existence of a sequence $\left(h_{n}\right)$ such that

$$
\frac{\nabla L^{r}\left(\nabla u^{r}\left(x+h_{n} e_{i}\right)\right)-\nabla L^{r}\left(\nabla u^{r}(x)\right)}{h_{n}} \rightarrow \frac{\mathrm{d}}{\mathrm{d} x_{i}} \nabla L^{r}\left(\nabla u^{r}\right)
$$

so that the left hand side of (3.4) converges to $\int_{\Omega}\left\langle\frac{\mathrm{d}}{\mathrm{d} x_{s}} \nabla L^{r}\left(\nabla u^{r}\right), \nabla \phi\right\rangle$.

We also have

$$
\begin{gathered}
\int_{\Omega} g_{u}\left(x, u^{r}(x)\right) \frac{\phi\left(x-h e_{s}\right)-\phi(x)}{-h} \mathrm{~d} x=\int_{0}^{1} \int_{\operatorname{supp}(\phi)+t h e_{s}} g_{u}\left(x, u^{r}(x)\right) \phi_{x_{s}}\left(x-t h e_{s}\right) \mathrm{d} x \mathrm{~d} t \\
=\int_{0}^{1} \int_{\operatorname{supp}(\phi)} g_{u}\left(x+t h e_{s}, u^{r}\left(x+t h e_{s}\right)\right) \phi_{x_{s}}(x) \mathrm{d} x \mathrm{~d} t \\
=\int_{\Omega} g_{u}\left(x, u^{r}(x)\right) \phi_{x_{s}}(x) \mathrm{d} x+\int_{0}^{1} \int_{\Omega}\left[g_{u}\left(x+h e_{s}, u^{r}\left(x+h e_{s}\right)\right)-g_{u}\left(x, u^{r}(x)\right] \phi_{x_{s}}(x) \mathrm{d} x \mathrm{~d} t .\right.
\end{gathered}
$$

By Assumption 2.1, ii), we obtain that the map $x \rightarrow g_{u}\left(x, u^{r}(x)\right)$ is in $L_{\mathrm{loc}}^{2}(\Omega)$, so that $\| g_{u}\left(\cdot+h e_{s}, u^{r}\left(\cdot+h e_{s}\right)\right)-$ $g_{u}\left(\cdot, u^{r}(\cdot)\right) \|_{L^{2}(\operatorname{supp}(\phi))} \rightarrow 0$, thus proving the lemma. 
Lemma 3.2. There exists $K$, depending neither on $r$ nor on $p$, such that $\left\|\nabla u^{r}\right\|_{L^{2}(\Omega)} \leq K$ and $\left\|u^{r}\right\|_{L^{2}(\Omega)} \leq K$.

Proof. Set $L^{0}(\xi)=\frac{1}{2}|\xi|^{2}+1$, so that, for any $1<p<2$ and any $r \leq 1$, we have $L^{r}(\xi) \leq L^{0}(\xi)+1$.

Let $u^{0}$ be a solution to the problem of minimizing

$$
\int_{\Omega}\left[L^{0}(\nabla v(x))+1+g(x, v(x))\right] \mathrm{d} x \quad \text { on } \quad w^{0}+W_{0}^{1,2}(\Omega)
$$

and set $V=\int_{\Omega}\left[L^{0}\left(\nabla u^{0}(x)\right)+1+g\left(x, u^{0}(x)\right)\right] \mathrm{d} x$. Then

$$
V \geq \int_{\Omega}\left[L^{r}\left(\nabla u^{0}\right)+g\left(x, u^{0}\right)\right] \geq \int_{\Omega}\left[L^{r}\left(\nabla u^{r}\right)+g\left(x, u^{r}\right)\right] \geq \int_{\Omega}\left[\frac{1}{2}\left|\nabla u^{r}\right|^{2}+g\left(x, u^{r}\right)\right] ;
$$

on the other hand, recalling Assumption 2.1, for a constant $\alpha$ to be fixed, from $\int \lambda_{g}|u| \leq \frac{1}{2} \alpha^{2} \int\left(\lambda_{g}\right)^{2}+\frac{1}{2} \frac{1}{\alpha^{2}} \int|u|^{2}$ we obtain

$$
\int_{\Omega} g\left(x, u^{r}(x)\right) \mathrm{d} x \geq \int \tau-\frac{1}{2} \alpha^{2} \int\left(\lambda_{g}\right)^{2}-\frac{1}{2} \frac{1}{\alpha^{2}} \int\left|u^{r}\right|^{2} .
$$

Call $P$ the Poincaré constant in $W^{1,2}(\Omega)$; from $\int\left|u^{r}\right|^{2}=\int\left|w^{0}-\left(u^{r}-w^{0}\right)\right|^{2} \leq 2 \int\left|w^{0}\right|^{2}+2 P \int\left|\nabla\left(u^{r}-w^{0}\right)\right|^{2} \leq$ $2 \int\left|w^{0}\right|^{2}+4 P \int\left|\nabla u^{r}\right|^{2}+4 P \int\left|\nabla w^{0}\right|^{2}$, we obtain

$$
\int_{\Omega} g\left(x, u^{r}\right) \geq \int \tau-\frac{1}{2} \alpha^{2} \int\left(\lambda_{g}\right)^{2}-\frac{1}{2} \frac{1}{\alpha^{2}}\left[4 P \int\left|\nabla u^{r}\right|^{2}+\int\left|\nabla w^{0}\right|^{2}(2+4 P)\right] .
$$

Hence,

$$
\int_{\Omega} \frac{1}{2}\left|\nabla u^{r}\right|^{2} \leq V-\int_{\Omega} g\left(x, u^{r}\right) \leq V-\int \tau+\frac{1}{2} \alpha^{2} \int\left(\lambda_{g}\right)^{2}+\frac{2 P}{\alpha^{2}} \int\left|\nabla u^{r}\right|^{2}+\frac{1}{2 \alpha^{2}} \int\left|\nabla w^{0}\right|^{2}(2+4 P) .
$$

Choose $\alpha$ such that $\frac{2 P}{\alpha^{2}}=\frac{1}{4}$ to obtain $\int\left|\nabla u^{r}\right|^{2} \leq 4\left[V+\int\left(-\tau+\frac{1}{2} \alpha^{2}\left(\lambda_{g}\right)^{2}+\frac{2+4 P}{2 \alpha^{2}}\left|\nabla w^{0}\right|^{2}\right)\right]=k_{1}$.

From this, making use of $w^{0} \in W^{1,2}$ and of Poincaré's inequality, we infer that for some $k_{2}$, we also have $\int_{\Omega}\left|u^{r}\right|^{2} \leq k_{2}$ for all $r \leq 1$.

A similar estimate was proved in [4].

Proof of Theorem 2.2.

a) Consider the function

$$
\gamma_{r}(t)=\frac{l_{r}^{\prime}(t)}{t}= \begin{cases}r^{p-2} & \text { for }|t| \leq r \\ |t|^{p-2} & \text { for } r<|t|<1 \\ 1 & \text { otherwise }\end{cases}
$$

then, as in the Proof of Lemma 3.1, the map $x \rightarrow \gamma_{r}\left(\left|\nabla u^{r}(x)\right|\right)$ is in $W_{l o c}^{1,2}$ and

$$
\frac{\mathrm{d}}{\mathrm{d} x_{s}} \gamma_{r}\left(\left|\nabla u^{r}(x)\right|\right)= \begin{cases}0 & \text { for }\left|\nabla u^{r}\right| \leq r \text { or }\left|\nabla u^{r}\right| \geq 1 \\ (p-2)\left|\nabla u^{r}\right|^{p-3}\left\langle\frac{\nabla u^{r}}{\left|\nabla u^{r}\right|}, \nabla u_{x_{s}}^{r}\right\rangle & \text { for } r<\left|\nabla u^{r}\right|<1 .\end{cases}
$$

Moreover, $1 \leq \gamma_{r} \leq r^{p-2}$ and $\left|\frac{\mathrm{d}}{\mathrm{d} x_{s}} \gamma_{r}\left(\left|\nabla u^{r}\right|\right)\right| \leq(2-p) r^{p-3}\left|\nabla u_{x_{s}}^{r}\right|$. Then, the map $x \rightarrow \gamma_{r}\left(\left|\nabla u^{r}(x)\right|\right) u_{x_{i}}(x)$ is in $W_{\text {loc }}^{1,2}(\Omega)$ and, setting $H_{u^{r}}$ to be the Hessian matrix of $u^{r}$, we obtain

$$
\nabla\left(\gamma_{r}\left(\left|\nabla u^{r}\right|\right) u_{x_{i}}^{r}\right)= \begin{cases}\gamma_{r}\left(\left|\nabla u^{r}\right|\right) \nabla u_{x_{i}}^{r} & \text { for }\left|\nabla u^{r}\right| \leq r \text { or }\left|\nabla u^{r}\right| \geq 1 \\ (p-2)\left|\nabla u^{r}\right|^{p-2} H_{u^{r}} \frac{\nabla u^{r}}{\left|\nabla u^{r}\right|} \frac{u_{x_{i}}^{r}}{\left|\nabla u^{r}\right|}+\gamma_{r}\left(\left|\nabla u^{r}\right|\right) \nabla u_{x_{i}}^{r} & \text { for } r \leq\left|\nabla u^{r}\right| \leq 1 .\end{cases}
$$


b) Let $x^{0}$ and $\delta^{0}$ be such that $B\left(x^{0}, 4 \delta^{0}\right) \subset \subset \Omega$. Let $\eta \in C_{0}^{\infty}\left(B\left(x^{0}, 2 \delta^{0}\right)\right)$ be such that $0 \leq \eta \leq 1$ and that $\eta(x)=1$ for $x \in B\left(x^{0}, \delta^{0}\right)$; we recall that $D_{\eta}=\sup \{|\nabla \eta(x)|\}$. Then, the function $\phi=\left[\eta^{2} \gamma_{r}\left(\left|\nabla u^{r}\right|\right) u_{x_{i}}^{r}\right]$ is in $W_{0}^{1,2}\left(B\left(x^{0}, 3 \delta^{0}\right)\right)$ and from Lemma 3.1 we have

$$
\int_{\Omega}\left\langle\frac{\mathrm{d}}{\mathrm{d} x_{i}} \frac{l_{r}^{\prime}\left(\left|\nabla u^{r}\right|\right)}{\left|\nabla u^{r}\right|} \nabla u^{r}, \nabla \phi\right\rangle=\int_{\Omega} g_{u}\left(\cdot, u^{r}\right) \phi_{x_{i}}
$$

i.e.,

$$
\begin{aligned}
& \int_{B\left(x^{0}, 3 \delta^{0}\right)}\left\langle\frac{l_{r}^{\prime}\left(\left|\nabla u^{r}\right|\right)}{\left|\nabla u^{r}\right|} \nabla u_{x_{i}}^{r}+\left(\frac{\mathrm{d}}{\mathrm{d} x_{i}} \frac{l_{r}^{\prime}\left(\left|\nabla u^{r}\right|\right)}{\left|\nabla u^{r}\right|}\right) \nabla u^{r}, \quad 2 \eta \nabla \eta \gamma_{r}\left(\left|\nabla u^{r}\right|\right) u_{x_{i}}^{r}+\eta^{2} \nabla\left(\gamma_{r}\left(\left|\nabla u^{r}\right|\right) u_{x_{i}}\right)\right\rangle \mathrm{d} x \\
& =\int_{B\left(x^{0}, 3 \delta^{0}\right)} g_{u}\left(\cdot, u^{r}\right)\left[2 \eta \eta_{x_{i}} \gamma_{r}\left(\left|\nabla u^{r}\right|\right) u_{x_{i}}^{r}+\eta^{2} \frac{\mathrm{d}}{\mathrm{d} x_{i}}\left(\gamma_{r}\left(\left|\nabla u^{r}\right|\right) u_{x_{i}}^{r}\right)\right] \mathrm{d} x
\end{aligned}
$$

We shall call $G_{i}$ the term at the right hand side.

Since the above equality holds for every $i$, we obtain

$$
\begin{aligned}
& \sum_{i} \int_{B\left(x^{0}, 3 \delta^{0}\right)}\left\langle\frac{\mathrm{d}}{\mathrm{d} x_{i}} \frac{l_{r}^{\prime}\left(\left|\nabla u^{r}\right|\right)}{\left|\nabla u^{r}\right|} \nabla u^{r}, \eta^{2} \nabla\left(\gamma_{r}\left(\left|\nabla u^{r}\right|\right) u_{x_{i}}^{r}\right)\right\rangle \mathrm{d} x \\
& \leq \sum_{i}\left|\int_{B\left(x^{0}, 3 \delta^{0}\right)}\left\langle\frac{\mathrm{d}}{\mathrm{d} x_{i}} \frac{l_{r}^{\prime}\left(\left|\nabla u^{r}\right|\right)}{\left|\nabla u^{r}\right|} \nabla u^{r}, 2 \eta \nabla \eta \gamma_{r}\left(\left|\nabla u^{r}\right|\right) u_{x_{i}}^{r}\right\rangle\right| \mathrm{d} x+\sum_{i} G_{i}
\end{aligned}
$$

c) For $j=1, \ldots, N$, we have

$$
\begin{gathered}
\left(\nabla_{x}\left(\frac{l_{r}^{\prime}\left(\left|\nabla u^{r}\right|\right)}{\left|\nabla u^{r}\right|} \nabla u^{r}\right)\right)_{i, j}=\frac{l_{r}^{\prime}\left(\left|\nabla u^{r}\right|\right)}{\left|\nabla u^{r}\right|} u_{x_{j} x_{i}}^{r}+\left(\frac{\mathrm{d}}{\mathrm{d} x_{i}} \frac{l_{r}^{\prime}\left(\left|\nabla u^{r}\right|\right)}{\left|\nabla u^{r}\right|}\right) u_{x_{j}}^{r} \\
=\frac{l_{r}^{\prime}}{\left|\nabla u^{r}\right|} u_{x_{j} x_{i}}^{r}+\left\langle\frac{\nabla u^{r}}{\left|\nabla u^{r}\right|}, \nabla u_{x_{i}}^{r}\right\rangle\left(l_{r}^{\prime \prime}-\frac{l_{r}^{\prime}}{\left|\nabla u^{r}\right|}\right) \frac{u_{x_{j}}^{r}}{\left|\nabla u^{r}\right|}
\end{gathered}
$$

i.e.,

and we obtain

$$
\nabla_{x}\left(\frac{l_{r}^{\prime}\left(\left|\nabla u^{r}\right|\right)}{\left|\nabla u^{r}\right|} \nabla u^{r}\right)=\frac{l_{r}^{\prime}}{\left|\nabla u^{r}\right|} H_{u^{r}}+\left(l_{r}^{\prime \prime}-\frac{l_{r}^{\prime}}{\left|\nabla u^{r}\right|}\right)\left(\frac{\nabla u^{r}}{\left|\nabla u^{r}\right|} H_{u^{r}}\right) \otimes \frac{\nabla u^{r}}{\left|\nabla u^{r}\right|}
$$

$$
\begin{gathered}
\left|\nabla_{x}\left(\frac{l_{r}^{\prime}\left(\left|\nabla u^{r}\right|\right)}{\left|\nabla u^{r}\right|} \nabla u^{r}\right)\right|^{2} \\
=\left(\frac{l_{r}^{\prime}}{\left|\nabla u^{r}\right|}\right)^{2}\left|H_{u^{r}}\right|^{2}+\left(l_{r}^{\prime \prime}-\frac{l_{r}^{\prime}}{\left|\nabla u^{r}\right|}\right)^{2}\left|\frac{\nabla u^{r}}{\left|\nabla u^{r}\right|} H_{u^{r}}\right|^{2}+2\left(l_{r}^{\prime \prime}-\frac{l_{r}^{\prime}}{\left|\nabla u^{r}\right|}\right) \frac{l_{r}^{\prime}}{\left|\nabla u^{r}\right|}\left|\frac{\nabla u^{r}}{\left|\nabla u^{r}\right|} H_{u^{r}}\right|^{2} \\
=\left(\frac{l_{r}^{\prime}}{\left|\nabla u^{r}\right|}\right)^{2}\left|H_{u^{r}}\right|^{2}+\left(\left(l_{r}^{\prime \prime}\right)^{2}-\left(\frac{l_{r}^{\prime}}{\left|\nabla u^{r}\right|}\right)^{2}\right)\left|\frac{\nabla u^{r}}{\left|\nabla u^{r}\right|} H_{u^{r}}\right|^{2}
\end{gathered}
$$

so that

$$
\inf \left\{\left(l_{r}^{\prime \prime}\right)^{2},\left(\frac{l_{r}^{\prime}}{\left|\nabla u^{r}\right|}\right)^{2}\right\}\left|H_{u^{r}}\right|^{2} \leq\left|\nabla_{x}\left(\frac{l_{r}^{\prime}\left(\left|\nabla u^{r}\right|\right)}{\left|\nabla u^{r}\right|} \nabla u^{r}\right)\right|^{2} \leq \sup \left\{\left(l_{r}^{\prime \prime}\right)^{2},\left(\frac{l_{r}^{\prime}}{\left|\nabla u^{r}\right|}\right)^{2}\right\}\left|H_{u^{r}}\right|^{2} .
$$

We also have, computing $l_{r}^{\prime}, l_{r}^{\prime \prime}, \gamma_{r}$ and $\gamma_{r}^{\prime}$ at $\left|\nabla u^{r}\right|$,

$$
\sum_{i}\left\langle\frac{\mathrm{d}}{\mathrm{d} x_{i}} \frac{l_{r}^{\prime}\left(\left|\nabla u^{r}\right|\right)}{\left|\nabla u^{r}\right|} \nabla u^{r}, \nabla\left(\gamma_{r} u_{x_{i}}^{r}\right)\right\rangle
$$




$$
\begin{aligned}
& =\sum_{i} \sum_{j}\left(\frac{l_{r}^{\prime}}{\left|\nabla u^{r}\right|} u_{x_{j} x_{i}}^{r}+\left(\sum_{s} \frac{u_{x_{s}}^{r}}{\left|\nabla u^{r}\right|} u_{x_{i} x_{s}}^{r}\right)\left(l_{r}^{\prime \prime}-\frac{l_{r}^{\prime}}{\left|\nabla u^{r}\right|}\right) \frac{u_{x_{j}}^{r}}{\left|\nabla u^{r}\right|}\right) \\
& \cdot\left(\gamma_{r} u_{x_{j} x_{i}}^{r}+\left(\gamma_{r}\right)^{\prime}\left(\sum_{l} u_{x_{j} x_{l}}^{r} \frac{u_{x_{l}}^{r}}{\left|\nabla u^{r}\right|}\right) u_{x_{i}}^{r}\right) \\
& =\frac{l_{r}^{\prime}}{\left|\nabla u^{r}\right|} \gamma_{r}\left|H_{u^{r}}\right|^{2}+\left(l_{r}^{\prime \prime}-\frac{l_{r}^{\prime}}{\left|\nabla u^{r}\right|}\right) \gamma_{r}^{\prime}\left|\nabla u^{r}\right|\left(\sum_{i, s} \frac{u_{x_{i}}^{r}}{\left|\nabla u^{r}\right|} \frac{u_{x_{s}}^{r}}{\left|\nabla u^{r}\right|} u_{x_{i} x_{s}}^{r}\right) \\
& \left(\sum_{j, l} \frac{u_{x_{j}}^{r}}{\left|\nabla u^{r}\right|} \frac{u_{x_{l}}^{r}}{\left|\nabla u^{r}\right|} u_{x_{j} x_{l}}^{r}\right) \\
& +\gamma_{r}\left(l_{r}^{\prime \prime}-\frac{l_{r}^{\prime}}{\left|\nabla u^{r}\right|}\right) \sum_{i}\left(\sum_{j} \frac{u_{x_{j}}^{r}}{\left|\nabla u^{r}\right|} u_{x_{j} x_{i}}^{r}\right)\left(\sum_{s} \frac{u_{x_{s}}^{r}}{\left|\nabla u^{r}\right|} u_{x_{i} x_{s}}^{r}\right) \\
& +\frac{l_{r}^{\prime}}{\left|\nabla u^{r}\right|} \gamma_{r}^{\prime}\left|\nabla u^{r}\right|\left(H_{u^{r}} \frac{\nabla u^{r}}{\left|\nabla u^{r}\right|}\right)^{2} \\
& =\frac{l_{r}^{\prime}}{\left|\nabla u^{r}\right|} \gamma_{r}\left|H_{u^{r}}\right|^{2}+\left(l_{r}^{\prime \prime}-\frac{l_{r}^{\prime}}{\left|\nabla u^{r}\right|}\right) \gamma_{r}^{\prime}\left|\nabla u^{r}\right|\left(\frac{\nabla u^{r} T}{\left|\nabla u^{r}\right|} H_{u^{r}} \frac{\nabla u^{r}}{\left|\nabla u^{r}\right|}\right)^{2} \\
& +\gamma_{r}\left(l_{r}^{\prime \prime}-\frac{l_{r}^{\prime}}{\left|\nabla u^{r}\right|}\right)\left|H_{u^{r}} \frac{\nabla u^{r}}{\left|\nabla u^{r}\right|}\right|^{2}+\frac{l_{r}^{\prime}}{\left|\nabla u^{r}\right|} \gamma_{r}^{\prime}\left|\nabla u^{r}\right|\left|H_{u^{r}} \frac{\nabla u^{r}}{\left|\nabla u^{r}\right|}\right|^{2} \\
& =\frac{l_{r}^{\prime}}{\left|\nabla u^{r}\right|} \gamma_{r}\left|H_{u^{r}}\right|^{2}+\left(l_{r}^{\prime \prime}-\frac{l_{r}^{\prime}}{\left|\nabla u^{r}\right|}\right) \gamma_{r}\left|H_{u^{r}} \frac{\nabla u^{r}}{\left|\nabla u^{r}\right|}\right|^{2} \\
& +\frac{l_{r}^{\prime}}{\left|\nabla u^{r}\right|} \gamma_{r}^{\prime}\left|\nabla u^{r}\right|\left[\left|H_{u^{r}} \frac{\nabla u^{r}}{\left|\nabla u^{r}\right|}\right|^{2}-\left(\frac{\nabla u^{r} T}{\left|\nabla u^{r}\right|} H_{u^{r}} \frac{\nabla u^{r}}{\left|\nabla u^{r}\right|}\right)^{2}\right] \\
& +l_{r}^{\prime \prime} \gamma_{r}^{\prime}\left|\nabla u^{r}\right|\left({\frac{\nabla u^{r}}{\left|\nabla u^{r}\right|}}^{T} H_{u^{r}} \frac{\nabla u^{r}}{\left|\nabla u^{r}\right|}\right)^{2} \\
& \geq \gamma_{r}\left[\frac{l_{r}^{\prime}}{\left|\nabla u^{r}\right|}\left|H_{u^{r}}\right|^{2}+\left(l_{r}^{\prime \prime}-\frac{l_{r}^{\prime}}{\left|\nabla u^{r}\right|}\right)\left|H_{u^{r}} \frac{\nabla u^{r}}{\left|\nabla u^{r}\right|}\right|^{2}\right] \\
& \geq \gamma_{r}\left[\inf \left\{l_{r}^{\prime \prime}, \frac{l_{r}^{\prime}}{\left|\nabla u^{r}\right|}\right\}\right]\left|H_{u^{r}}\right|^{2}
\end{aligned}
$$

where

$$
\gamma_{r}(t) \inf \left\{l_{r}^{\prime \prime}(t), \frac{l_{r}^{\prime}(t)}{t}\right\}= \begin{cases}r^{2(p-2)} & \text { for }|t| \leq r \\ (p-1)|t|^{2(p-2)} & \text { for } r \leq|t| \leq 1 \\ 1 & \text { otherwise. }\end{cases}
$$

Hence we have obtained

$$
\int_{B\left(x^{0}, 3 \delta^{0}\right)} \eta^{2} \gamma_{r}\left[\inf \left\{l_{r}^{\prime \prime}, \frac{l_{r}^{\prime}}{\left|\nabla u^{r}\right|}\right\}\right]\left|H_{u^{r}}\right|^{2} \mathrm{~d} x \leq \sum_{i} \int_{B\left(x^{0}, 3 \delta^{0}\right)} \eta^{2}\left\langle\frac{\mathrm{d}}{\mathrm{d} x_{i}} \frac{l_{r}^{\prime}\left(\left|\nabla u^{r}\right|\right)}{\left|\nabla u^{r}\right|^{r}} \nabla u^{r}, \nabla\left(\gamma_{r}\left(\left|\nabla u^{r}\right|\right) u_{x_{i}}\right)\right\rangle \mathrm{d} x
$$


so that, from (3.8),

$$
\begin{aligned}
\int_{B\left(x^{0}, 3 \delta^{0}\right)} \eta^{2} \gamma_{r}\left[\inf \left\{l_{r}^{\prime \prime}, \frac{l_{r}^{\prime}}{\left|\nabla u^{r}\right|}\right\}\right] & \left|H_{u^{r}}\right|^{2} \mathrm{~d} x \\
& \leq \sum_{i}\left|\int_{B\left(x^{0}, 3 \delta^{0}\right)}\left\langle\frac{\mathrm{d}}{\mathrm{d} x_{i}} \frac{l_{r}^{\prime}\left(\left|\nabla u^{r}\right|\right)}{\left|\nabla u^{r}\right|} \nabla u^{r}, 2 \eta \nabla \eta \gamma_{r}\left(\left|\nabla u^{r}\right|\right) u_{x_{i}}^{r}\right\rangle\right| \mathrm{d} x+\sum_{i} G_{i}
\end{aligned}
$$

d) From (3.6) we have that

$$
\begin{aligned}
\sum_{i} G_{i}= & \int_{B\left(x^{0}, 3 \delta^{0}\right)}\left[g_{u}\left(x, u^{r}\right) 2 \eta \gamma_{r}\left(\left|\nabla u^{r}\right|\right)\left\langle\nabla \eta, \nabla u^{r}\right\rangle+\eta^{2} g_{u}\left(x, u^{r}\right) \Delta u^{r}\right] \mathrm{d} x \\
& +\int_{B\left(x^{0}, 3 \delta^{0}\right) \cap\left\{r \leq\left|\nabla u^{r}(x)\right| \leq 1\right\}} g_{u}\left(x, u^{r}\right) \eta^{2}(p-2)\left|\nabla u^{r}\right|^{p-2}\left(\frac{\nabla u^{r}}{\left|\nabla u^{r}\right|}\right)^{T} H_{u^{r}} \frac{\nabla u^{r}}{\left|\nabla u^{r}\right|} \mathrm{d} x \\
& \leq \int_{B\left(x^{0}, 3 \delta^{0}\right)}\left[\eta^{2} g_{u}^{2}+|\nabla \eta|^{2}+\frac{4}{p-1} \eta^{2} g_{u}^{2}+\frac{p-1}{4} \eta^{2}\left|H_{u^{r}}\right|^{2}\right] \mathrm{d} x \\
& +\int_{B\left(x^{0}, 3 \delta^{0}\right) \cap\left\{r \leq\left|\nabla u^{r}(x)\right| \leq 1\right\}}\left[\frac{4}{p-1} \eta^{2} g_{u}^{2}+\eta^{2} \frac{p-1}{4}\left|\nabla u^{r}\right|^{2(p-2)}\left|H_{u^{r}}\right|^{2}\right] \mathrm{d} x
\end{aligned}
$$

By Assumption 2.1, $g_{u}\left(x, u^{r}\right)^{2} \leq 2\left[\left(\lambda_{2}\right)^{2}+\left(\lambda_{\infty}\left|u^{r}\right|\right)^{2}\right]$; hence, applying Lemma 3.2 we infer that there exists a constant $K^{0}$, independent of $r$ and $p$, such that the right hand side of (3.11) is bounded above by

$$
\frac{K^{0}}{p-1}+\frac{1}{2} \int_{B\left(x^{0}, 3 \delta^{0}\right)} \eta^{2}(p-1)\left|\nabla u^{r}\right|^{2(p-2)}\left|H_{u^{r}}\right|^{2} \mathrm{~d} x .
$$

Then, from $\inf \left\{l_{r}^{\prime \prime}, \frac{l_{r}^{\prime}}{\left|\nabla u^{r}\right|}\right\} \geq p-1,(3.10)$ gives

$$
\begin{aligned}
& \frac{1}{2} \int_{B\left(x^{0}, 3 \delta^{0}\right)} \eta^{2} \gamma_{r}\left[\inf \left\{l_{r}^{\prime \prime}, \frac{l_{r}^{\prime}}{\left|\nabla u^{r}\right|}\right\}\right]\left|H_{u^{r}}\right|^{2} \mathrm{~d} x \\
& \leq \frac{1}{p-1} K^{0}+\sum_{i}\left|\int_{B\left(x^{0}, 3 \delta^{0}\right)}\left\langle\frac{\mathrm{d}}{\mathrm{d} x_{i}} \frac{l_{r}^{\prime}\left(\left|\nabla u^{r}\right|\right)}{\left|\nabla u^{r}\right|} \nabla u^{r}, 2 \eta \nabla \eta \gamma_{r}\left(\left|\nabla u^{r}\right|\right) u_{x_{i}}^{r}\right\rangle\right| \mathrm{d} x \\
& \leq \frac{1}{p-1} K^{0}+\int_{B\left(x^{0}, 3 \delta^{0}\right)}\left[\frac{p-1}{4}\left|\nabla_{x}\left(\nabla L\left(\nabla u^{r}\right)\right)\right|^{2} \eta^{2}+\frac{4}{p-1} N|\nabla \eta|^{2}\right] \mathrm{d} x \\
& \leq \frac{1}{p-1} K^{0}+\int_{B\left(x^{0}, 3 \delta^{0}\right)}\left[\frac{1}{4} \gamma_{r} \inf \left\{l_{r}^{\prime \prime}, \frac{l_{r}^{\prime}}{|t|}\right\}\left|\nabla_{x}\left(\nabla L\left(\nabla u^{r}\right)\right)\right|^{2} \eta^{2}+\frac{4}{p-1} N|\nabla \eta|^{2}\right] \mathrm{d} x
\end{aligned}
$$

and we obtain

$$
\frac{p-1}{4} \int_{B\left(x^{0}, 3 \delta^{0}\right)} \eta^{2} \mid \nabla_{x}\left(\left.\nabla L_{r}\left(\nabla u^{r}\right)\right|^{2} \mathrm{~d} x \leq \frac{1}{4} \int_{B\left(x^{0}, 3 \delta^{0}\right)} \eta^{2} \gamma_{r}\left[\inf \left\{l_{r}^{\prime \prime}, \frac{l_{r}^{\prime}}{\left|\nabla u^{r}\right|}\right\}\right]\left|H_{u^{r}}\right|^{2} \mathrm{~d} x \leq \frac{K^{1}}{p-1} .\right.
$$

f) In particular,

$$
\int_{B\left(x^{0}, \delta^{0}\right)}\left|\nabla_{x} \nabla L_{r}\left(\nabla u^{r}\right)\right|^{2} \leq \frac{4}{(p-1)^{2}} K^{1}
$$

hence, the family $\left(\nabla_{x} \nabla L_{r}\left(\nabla u^{r}\right)\right)_{r}$ is bounded in $L^{2}\left(B\left(x^{0}, \delta^{0}\right)\right)$. The arbitrariness of $x^{0}$ and of $\delta^{0}$ then shows that, for every $\omega \subset \subset \Omega$, there exists $H$, independent of $r$ and $p$, such that $\left(\left\|\nabla_{x} \nabla L_{r}\left(\nabla u^{r}\right)\right\|_{L^{2}(\omega)}\right)_{r} \leq \frac{H}{(p-1)^{2}}$. Then, from (3.9) and since $\inf \left\{\left(l_{r}^{\prime \prime}\right)^{2},\left(\frac{l_{r}^{\prime}}{\left|\nabla u^{r}\right|}\right)^{2}\right\} \geq(p-1)^{2}$, we infer that

$$
\int_{\omega}\left|H_{u^{r}}\right|^{2} \leq \frac{1}{(p-1)^{2}} \int_{\omega}\left|\nabla_{x} \nabla L_{r}\left(\nabla u^{r}\right)\right|^{2} \leq \frac{1}{(p-1)^{4}} H^{2} .
$$


Then, we can assume that, for $s=1, \ldots, N$, there exists a sequence $\left(r^{n}\right)_{n}$ such that $\frac{\mathrm{d}}{\mathrm{d} x_{s}} \nabla L_{r_{n}}\left(\nabla u^{r_{n}}\right)$ converges weakly in $L^{2}(\omega)$ to some $d_{\lambda}$, that $\nabla L_{r_{n}}\left(\nabla u^{r_{n}}\right)$ converges in $L^{2}(\omega)$ to a function $\lambda$, that $u^{r_{n}} \rightarrow u$ and, finally, that $\nabla u^{r_{n}} \rightarrow \nabla u$ in $L^{2}(\omega)$.

g) We claim that:

i) $\lambda=\nabla L(\nabla u) ; d_{\lambda}=\frac{\mathrm{d}}{\mathrm{d} x_{s}} \nabla L(\nabla u)$ and

$$
\left\|\frac{\mathrm{d}}{\mathrm{d} x_{s}} \nabla L(\nabla u)\right\|_{L^{2}(\omega)} \leq \frac{1}{\left(p-1^{2}\right.} H
$$

ii) $u$ is a solution to the Euler Lagrange equation, i.e., that, for every $\eta \in C_{c}^{1}(\Omega)$,

$$
\int_{\Omega}\left[\langle\nabla L(\nabla u(x)), \nabla \eta(x)\rangle+g_{u}(x, u(x)) \eta(x)\right] \mathrm{d} x=0 .
$$

To prove the claim, notice that, possibly passing to a subsequence, we can assume that both $\nabla u^{r_{n}} \rightarrow \nabla u$ and $\nabla L_{r_{n}}\left(\nabla u^{r_{n}}\right) \rightarrow \lambda$ pointwise a.e.. Fix $x$ such that the above holds and fix $\varepsilon$. By the continuity of $\nabla L$, let $\delta$ be such that $|\nabla u(x)-\xi| \leq \delta$ implies $|\nabla L(\nabla u(x))-\nabla L(\xi)|<\frac{\varepsilon}{2}$; let $n$ be so large that both $\left|\nabla u^{r_{n}}(x)-\nabla u(x)\right|<\delta$, and $\left\|\nabla L_{r_{n}}-\nabla L\right\|_{C}<\frac{\varepsilon}{2}$. Hence, for $n$ large,

$$
\begin{gathered}
\left|\nabla L_{r_{n}}\left(\nabla u^{r_{n}}(x)\right)-\nabla L(\nabla u(x))\right| \\
\leq\left|\nabla L_{r_{n}}\left(\nabla u^{r_{n}}(x)\right)-\nabla L\left(\nabla u^{r_{n}}(x)\right)\right|+\left|\nabla L\left(\nabla u^{r_{n}}(x)\right)-\nabla L\left(\nabla u^{r_{n}}(x)\right)\right|<\varepsilon,
\end{gathered}
$$

so that $|\lambda(x)-\nabla L(\nabla u(x))| \leq \varepsilon$, and by the arbitrariness of $\varepsilon$, we obtain

$$
\lambda(x)=\nabla L(\nabla u(x)) .
$$

Moreover, $\nabla L_{r_{n}}\left(\nabla u^{r_{n}}\right) \rightarrow \lambda$ in $L^{2}(\omega)$ and $\frac{\mathrm{d}}{\mathrm{d} x_{s}} \nabla L_{r_{n}}\left(\nabla u^{r_{n}}\right) \rightarrow d_{\lambda}$ weakly, imply $d_{\lambda}=\frac{\mathrm{d}}{\mathrm{d} x_{s}} \nabla L(\nabla u)$, so that from $\left\|\frac{\mathrm{d}}{\mathrm{d} x_{s}} \nabla L_{r_{n}}\left(\nabla u^{r}\right)\right\|_{L^{2}(\omega)} \leq \frac{1}{(p-1)^{2}} H$ we obtain that $\left\|\frac{\mathrm{d}}{\mathrm{d} x_{s}} \nabla L(\nabla u)\right\|_{L^{2}(\omega)} \leq \frac{1}{(p-1)^{2}} H$, thus proving i).

To prove ii), fix $\eta \in C_{c}^{1}(\Omega)$. We have that

$$
\int_{\Omega}\left[\left\langle\nabla L_{r_{n}}\left(\nabla u^{r_{n}}(x)\right), \nabla \eta(x)\right\rangle+g_{u}\left(x, u^{r_{n}}(x)\right) \eta(x)\right] \mathrm{d} x=0
$$

since $u^{r_{n}} \rightarrow u$ in $L^{2}(\omega)$ and $\nabla L_{r_{n}}\left(\nabla u^{r_{n}}\right) \rightarrow \nabla L(\nabla u)$, we obtain

$$
\int_{\Omega}\left[\langle\nabla L(\nabla u(x)), \nabla \eta(x)\rangle+g_{u}(x, u(x)) \eta(x)\right] \mathrm{d} x=0 .
$$

Hence, we have obtained the existence of a solution $u$ to the Euler-Lagrange equation such that, for every $s=1, \ldots, N$,

$$
\frac{\mathrm{d}}{\mathrm{d} x_{s}} \nabla L(\nabla u)=\frac{\mathrm{d}}{\mathrm{d} x_{s}} \begin{cases}\frac{\nabla u}{|\nabla u|^{2-p}} & \text { for }|\nabla u| \leq 1 \\ \nabla u & \text { for }|\nabla u| \geq 1\end{cases}
$$

belongs to $L^{2}(\omega)$; in particular, for every $i=1, \ldots, N$, both $u_{x_{i}}$ and $\frac{u_{x_{i}}}{|\nabla u|^{2-p}}$ belong to $W_{\text {loc }}^{1,2}$. 


\section{REFERENCES}

[1] E. Acerbi and N. Fusco, Regularity for minimizers of non-quadratic functionals. The case $1<p<2$. J. Math. Anal. Appl. 140 (1989) 115-135.

[2] E. DiBenedetto, C1+ local regularity of weak solutions of degenerate elliptic equations. Nonlin. Anal. 7 (1983) $827-850$.

[3] L. Esposito and G. Mingione, Some remarks on the regularity of weak solutions of degenerate elliptic systems. Rev. Mat. Complut. 11 (1998) 203-219.

[4] A. Cellina, A case of regularity of solutions to non-regular problems. SIAM J. Control. Optim. 53 (2015) $2835-2845$.

[5] M. Colombo and A. Figalli, An excess-decay result for a class of degenerate elliptic equations. Discr. Contin. Dyn. Syst. Ser. $S 7$ (2014) 631-652.

[6] J.L. Lewis, Regularity of the derivatives of solutions to certain degenerate elliptic equations. Indiana Univ. Math. J. 32 (1983) 849-858.

[7] P. Tolksdorf, Regularity for a more general class of quasilinear elliptic equations. J. Differ. Eq. 51 (1984) 126-150.

[8] K.Ulhenbeck, Regularity for a class of non-linear elliptic systems. Acta Math. 138 (1977) 219-240. 